Revue

Revue de l'histoire des religions

del'histoire des religions

4| 2017

Les discours sur les religions dans l'Empire romain : regards croisés entre " païens ", "juifs " et « chrétiens »

\title{
Les discours sur les religions dans l'Empire romain
}

Avant-propos

Francesco Massa

\section{OpenEdition}

Journals

Édition électronique

URL : http://journals.openedition.org/rhr/8822

DOI : $10.4000 /$ rhr.8822

ISSN : 2105-2573

Éditeur

Armand Colin

Édition imprimée

Date de publication : 1 décembre 2017

Pagination : $587-592$

ISBN : 978-2-200-93128-5

ISSN : 0035-1423

Référence électronique

Francesco Massa, "Les discours sur les religions dans l'Empire romain », Revue de l'histoire des religions [En ligne], 4 | 2017, mis en ligne le 01 décembre 2017, consulté le 14 janvier 2021. URL http://journals.openedition.org/rhr/8822 ; DOI : https://doi.org/10.4000/rhr.8822 


\section{Les discours sur les religions dans l'Empire romain Avant-propos ${ }^{1}$}

L'enluminure médiévale reproduite en quatrième de couverture de ce fascicule de la Revue de l'histoire des religions - mais aussi, pour la commodité de la consultation, à la fin de notre avantpropos - est tirée d'un manuscrit illustré de 1376, conservé à la Bibliothèque nationale de France. Ce manuscrit contient la première traduction française du De civitate Dei d'Augustin. La traduction, qui date de 1371-1375, est l'œuvre de Raoul de Presles, juriste et maître des requêtes du roi sous le règne de Charles $\mathrm{V}^{2}$. Au centre de l'image, l'on peut voir deux figures divines, chacune sur un piédestal évoquant une base de statue antique. Cette position en hauteur vise cependant aussi à faire de ces figures l'objet d'une interrogation critique. Les deux idoles sont entourées de trois groupes de personnages : au centre six individus à genoux, le regard tourné vers les statues comme pour indiquer un état de recueillement et de prière ; à gauche et à droite de l'image d'autres

1. Les contributions réunies dans ce dossier ont été présentées lors de journées d'études organisées à la Maison méditerranéenne des sciences de l'homme d'Aixen-Provence les 9 et 10 février 2015 par le laboratoire d'excellence LabexMed (Les sciences humaines et sociales au cour de l'interdisciplinarité pour la Méditerranée) et l'UMR 7297 (TDMAM Textes et documents de la Méditerranée antique et médiévale). Elles sont issues de travaux réalisés dans le cadre du LabexMed portant la référence 10-LABX-0090 et ont bénéficié d'une aide de l'État au titre du Programme Investissements d'Avenir, Initiative d'Excellence d'Aix-Marseille Université $A * M I D E X$. - Que les deux institutions et en particulier leurs directrices, Emmanuèle Caire et Brigitte Marin, qui ont accepté de financer ce projet, trouvent ici l'expression de ma sincère reconnaissance.

2. Sur cette traduction voir La cité de Dieu de saint Augustin traduite par Raoul de Presles (1371-1375), livre I à III, édition du manuscrit BnF, fr. 22912, sous la direction d'Olivier Bertrand, Paris, Honoré Champion, 2013. 
personnages se tiennent debout et se distinguent des orants par leurs coiffures et leurs vêtements. Le regard qu'ils portent vers les statues suggère une tout autre posture : ces hommes semblent débattre du statut de ces idoles «païennes » que leur regard analyse. Ce sont ces chrétiens en train de discuter de la fausse religion des païens en prière devant leurs dieux qui constituent véritablement le sujet de cette enluminure.

Cette image nous invite à réfléchir sur deux aspects centraux du dossier publié ici, dans le cadre d'une enquête interrogeant les discours sur la pluralité religieuse à l'époque impériale romaine : d'une part, l'existence d'un débat interne à la communauté chrétienne quant à la nature de la religion des « autres »; d'autre part, le fait que nous soyons confrontés au regard d'un groupe particulier sur les autres groupes religieux établis dans les territoires de l'Empire romain.

Comme je viens de le mentionner, cette enluminure illustre la traduction d'une œuvre fondamentale d'Augustin, la Cité de Dieu, rédigée entre 413 et 417, dans le sillage de cet événement hautement traumatisant que fut le sac de Rome en 410 par les Goths d'Alaric. La Cité de Dieu est sans doute la dernière grande œuvre tardo-antique de polémique chrétienne contre le "paganisme» gréco-romain. Cette œuvre renferme l'ensemble du vocabulaire et des mots-clés du conflit entre « religions » qui aura marqué quatre siècles de rivalités religieuses entre «païens » et «chrétiens »: pietas contre impietas (II, 28), superstitio contre uera religio (VII, 16), le deorum falsorum cultus contre la Christiana religio (IV, 7). Au début du $\mathrm{v}^{\mathrm{e}}$ siècle, le terme pagani est définitivement entré dans le vocabulaire latin des auteurs chrétiens ${ }^{3}$.

En un certain sens, la Cité de Dieu représente l'aboutissement d'une réflexion théorique, initiée par les auteurs grecs et latins dès le $\mathrm{II}^{\mathrm{e}}$ siècle, qui interroge la religion des «autres » et, ce faisant, construit véritablement un savoir sur ces religions auxquelles le christianisme s'oppose, le «paganisme » et le «judaïsme». Dans le contexte d'un empire pluri-religieux et multiculturel, pour définir leur identité propre, les chrétiens se trouvaient confrontés à la nécessité de tracer, d'établir les frontières séparant leur propre religion des diverses formes religieuses existant dans les territoires romains.

3. Sur l'histoire du terme paganus voir Alan Cameron, The Last Pagans of Rome, Oxford-New York, Oxford University Press, 2011, p. 14-32. 
Les études réunies dans le présent dossier ne se limitent pas, cependant, à l' analyse des textes de controverse - genre littéraire qui apparaît dans l'Antiquité tardive et reflète précisément l'émergence d'un champ spécifique du savoir. Les controverses religieuses sont le fruit d'une dynamique de compétition entre des communautés religieuses différentes ${ }^{4}$. La perspective suivie ici se veut plus large, puisqu'il s'agit d'interroger les constructions intellectuelles et les dynamiques discursives à l'œuvre chez les auteurs tardo-antiques qui débattent de l'objet « religion ». À ce titre, il faut considérer de manière comparative les discours des intellectuels issus de différents horizons religieux, des discours qui peuvent parfois prendre la forme d'un dialogue, ou d'une compétition, voire d'un conflit ouvert.

Depuis les travaux de Peter Brown, les spécialistes s'accordent à considérer la religion comme l'une des composantes principales de ce que l'on appelle l'Antiquité tardive ${ }^{5}$, dans la mesure où elle a joué un rôle croissant dans la politique et la société de l'Empire romain et ses transformations durant cette période ${ }^{6}$. La nouvelle place que la dimension religieuse occupe au sein de l'Empire, notamment par le biais de la diffusion des diverses formes de christianisme, a conduit à l'émergence d'un nouveau discours sur la religion, initié par les auteurs chrétiens anciens : sous leur influence, une nouvelle rhétorique commence donc à se développer, rhétorique que reflète déjà la littérature apologétique chrétienne du $\mathrm{II}^{\mathrm{e}}$ siècle ${ }^{7}$. Ce nouveau discours sur la religion se fonde sur un vocabulaire qui, certes, puise dans les catégories grecques et romaines, mais qui refaçonne et resémantise ces catégories. Depuis une génération, plusieurs travaux ont entrepris d'analyser, de façon ponctuelle, les catégories

4. Voir, par exemple, Flavia Ruani éd., Les controverses religieuses en syriaque, Paris, Geuthner, 2016.

5. Voir Peter Brown, World of Late Antiquity: from Marcus Aurelius to Muhammad, London, Thames and Hudson, 1971 ; Id., Authority and the Sacred : Aspects of the Christianisation of the Roman World, Cambridge, Cambridge University Press, 1995. Pour une définition de la notion d'Antiquité tardive, voir Edward James, "The Rise and Function of the Concept 'Late Antiquity' », Journal of Late Antiquity 1, 1, 2008, p. 20-30.

6. Cf. Jörg Rüpke, From Jupiter to Christ. On the History of Religion in the Roman Imperial Period, Oxford, Oxford University Press, 2014.

7. Sur les stratégies rhétoriques visant à construire la distinction entre « païens » et «chrétiens », voir Averil Cameron, Christianity and the Rhetoric of Empire. The Development of Christian Discourse, Berkeley-Los Angeles-Oxford, University of California Press, 1991. 
fondatrices du discours religieux chrétien de l'Antiquité tardive, par exemple les notions de «paganisme » (hellènismos, paganitas,

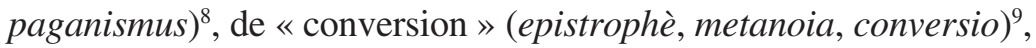

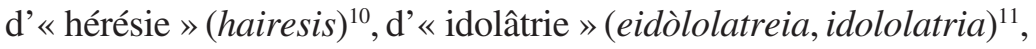
de «communauté » (koinònia, communitas) ${ }^{12}$, de "mystères» $(\text { mystèria })^{13}$, ou encore de « foi » et de « croyance » (pistis, fides) ${ }^{14}$.

Le processus par lequel les chrétiens ont élaboré un discours sur la religion implique une réflexion théorique sur cette notion, et la formation d'une nouvelle catégorie de « religion » dans les premiers siècles de notre ère (à partir des termes religio, eusebeia, thrèskeia). Des chercheurs tels que Maurice Sachot, Jonathan Z. Smith, Philippe Borgeaud, Jeremy M. Schott ou, plus récemment, Brent Nongbri, Daniel Boyarin et Carlin A. Barton, ont mis en évidence comment une nouvelle notion de « religion » est née dans l'Empire romain tardif ${ }^{15}$.

8. Voir Maijastina Kahlos, Debate and Dialogue. Christian and Pagan Culture, Aldershot, Ashgate, 2007.

9. Voir Annick Charles-Saget, «Sur quelques formes originaires de la constitution de soi », dans Id. éd., Retour, repentir et constitution de soi, Paris, Vrin, 1998, p. 13-36 ; Guy G. Stroumsa, La fin du sacrifice. Les mutations religieuses de l'Antiquité tardive, Paris, Odile Jacob, 2005, p. 42-47 ; Birgitte S. Bøgh éd., Conversion and Initiation in Antiquity: Shifting Identities, Creating Change, Frankfurt-York, P. Lang, 2014.

10. Voir Alain Le Boulluec, La notion d'hérésie dans la littérature grecque. $I I^{e}-$ III $^{e}$ siècles, Paris, Études Augustiniennes, 1985 et Enrico Norelli, « Costruzioni dell'eresia nel cristianesimo antico. Introduzione », Rivista di Storia del Cristianesimo 2, 2009, p. 323-332.

11. Voir Daniel Barbu, Naissance de l'idolâtrie: image, identité, religion, Liège, Presses Universitaires de Liège, 2016.

12. Voir Nicole Belayche, Simon C. Mimouni éd., Les communautés religieuses dans le monde gréco-romain. Essais de définition, Turnhout, Brepols, 2003 et Giovanni Filoramo, «Le peuple de Dieu. Comment naissent et fonctionnent les communautés de croyants? », dans Dionigi Albera, Katell Berthelot éd., Dieu, une enquête. Judaïsme, christianisme, islam, ce qui les distingue, ce qui les rapproche, Paris, Flammarion, 2013, p. 165-230.

13. Voir Francesco Massa, «La notion de 'mystères' au II siècle de notre ère : regards païens et Christian turn », dans Nicole Belayche, Francesco Massa éd., Les « cultes à mystères » : retour sur une catégorie, Mètis NS 14, 2016, p. 109-132.

14. Voir Jean Wirth, « La naissance du concept de croyance (XII-XVII ${ }^{\mathrm{e}}$ siècles) », Bibliothèque d'Humanisme et Renaissance, 45, 1, 1983, p. 7-58.

15. Voir Maurice Sachot, «Origine et trajectoire d'un mot : religion», Revue de philosophie ancienne, 21, 2, 2003, p. 3-32 ; Jonathan Z. Smith, «Religion, Religions, Religious », dans Id., Relating Religion. Essays in the Study of Religion, Chicago, 2004, p. 179-196 ; Jeremy M. Schott, Christianity, Empire, and the Making of Religion in Late Antiquity, Philadelphia, University of Pennsylvania Press, 2008 ; Philippe Borgeaud, L'histoire des religions, Lausanne, 2013 ; Brent Nongbri, Before Religion. A History of a Modern Concept, New Haven-London, 
Cette catégorie visait premièrement à séparer l'identité religieuse des individus des autres aspects de la vie sociale et politique.

Le présent dossier vise tout d'abord à tester cette hypothèse à partir des sources chrétiennes anciennes, et ensuite à contextualiser l'invention de cette nouvelle catégorie épistémologique dans le cadre plus large d'un empire multiculturel et pluri-religieux, au sein duquel les identités religieuses étaient loin d'être établies de manière tranchée. Mais on se gardera d'oublier que la réflexion chrétienne sur les religions est aussi dépendante d'une tradition intellectuelle qui, depuis les enquêtes ethnographiques d'Hérodote et les spéculations philosophiques de Platon, pose la question des origines des pratiques cultuelles, et réfléchit sur les savoirs religieux des « autres ». C'est pourquoi il convient de considérer de manière plus large le contexte culturel dans lequel les auteurs chrétiens ont pensé les religions des « autres », et de lire de manière comparatiste les discours des païens, des juifs et des chrétiens sur les croyances et les pratiques religieuses. Tous ces intellectuels partageaient une même culture, ou paideia, et sont le produit d'une même réalité historique et religieuse. Néanmoins, l'appartenance religieuse des uns et des autres a contribué à faire naître des réflexions différenciées, affirmant et construisant tout à la fois des systèmes de pensée et des modèles herméneutiques particuliers.

Grâce à un regard comparatiste sur les « discours » relatifs aux pratiques religieuses, on pourra éclairer si, et si oui comment, les auteurs païens, juifs et chrétiens ont construit une certaine manière de penser l'histoire comparée des religions, et ont modélisé à l'époque tardive « l'histoire des religions » comme objet de réflexion. Il s' agit de se demander quels types de questions ils se sont posé et quels étaient leurs objectifs. Ces questions sont d'autant plus importantes si l'on considère que les modèles interprétatifs construits dans l'Antiquité tardive ont servi de terreau aux réflexions des penseurs et des savants de l'Europe moderne ayant consacré leurs travaux à l'histoire des religions.

Francesco.Massa@unige.ch

Yale University Press, 2013; Carlin A. Barton, Daniel Boyarin, Imagine No Religion. How Modern Abstractions Hide Ancient Realities, New York, Fordham University Press, 2016. 


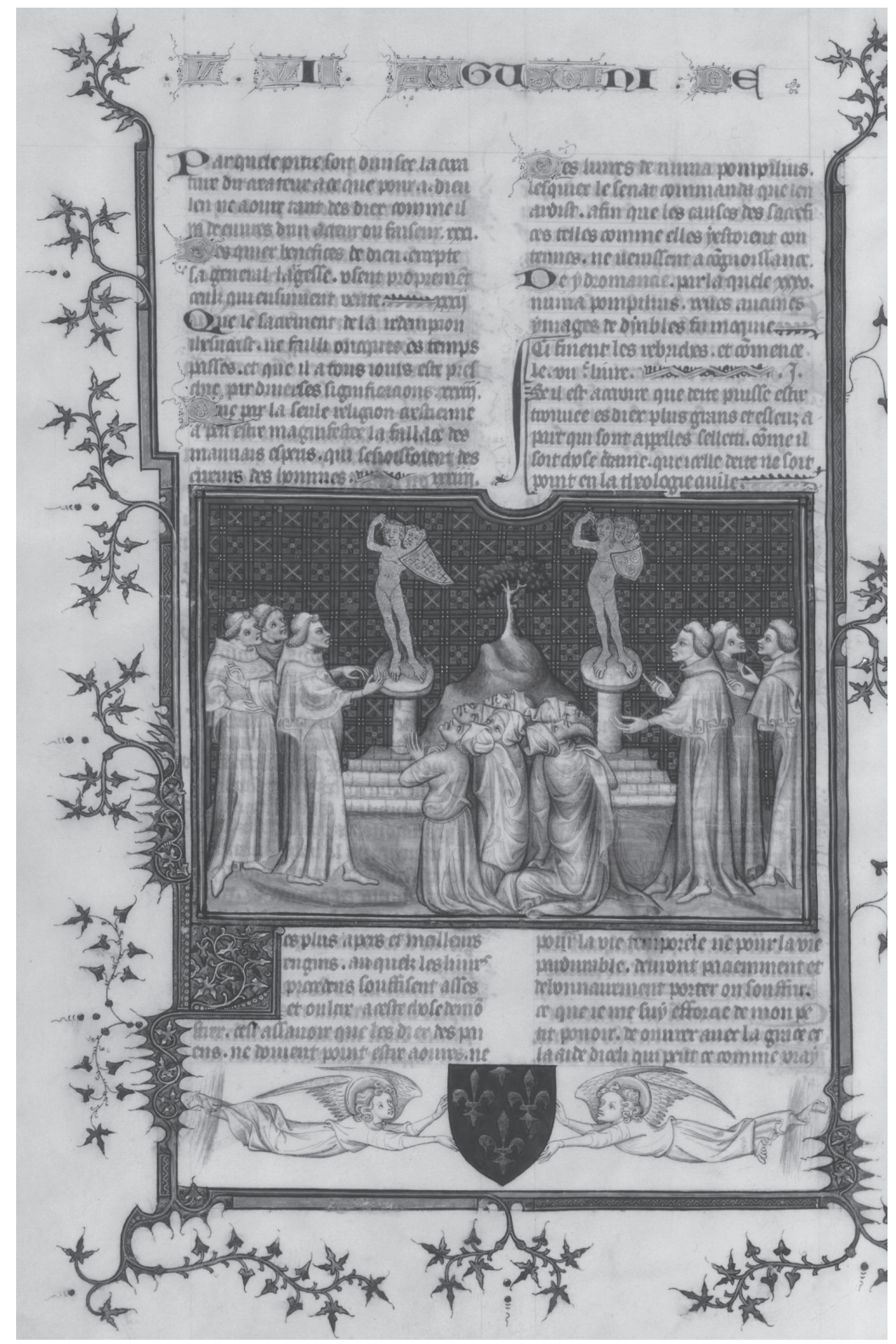

De la nature des dieux

Saint Augustin, La Cité de Dieu

(C) Bibliothèque nationale de France

Manuscrits occidentaux, Français 22912 [1376], fol. 303v 